\title{
Comportamento de Sterculia pruriens (Aubl.) Schum. em floresta tropical manejada em Moju-PA
}

\author{
Fernando Cristóvam da Silva JARDIM ${ }^{1}$, Marcelo da Silva SOARES ${ }^{2}$ \\ RESUMO \\ Foi avaliado o crescimento diamétrico de uma população de Sterculia pruriens (Aublet) Schumann (axixá) após exploração \\ florestal seletiva, na Estação Experimental da Embrapa Amazônia Oriental, Moju, Pará, localizada entre as coordenadas

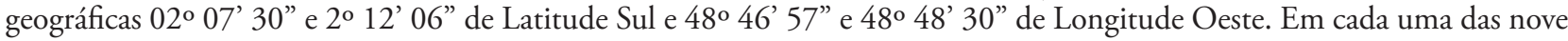 \\ clareiras selecionadas foram instaladas faixas de $10 \mathrm{~m}$ x 50m, começando na bordadura da clareira para o interior da floresta, \\ nas direçôes Norte, Sul, Este e Oeste. Cada faixa foi dividida em parcelas quadradas de $10 \mathrm{~m}$ de lado, que foram numeradas \\ de 1 a 5 da borda para o interior da floresta e constituem o nível II de abordagem. Foi analisado a distribuição diamétrica \\ da espécie com amplitude de DAP $\geq 5 \mathrm{~cm}$ em intervalos de $5 \mathrm{~cm}$ de diâmetro, até o valor máximo de $66,8 \mathrm{~cm}$ encontrado no \\ início do monitoramento. $\mathrm{Na}$ área de estudo foi encontrado densidade elevada da espécie, logo após a exploração florestal \\ e após três anos desta. Ao final do estudo, foi observado um acréscimo de 9,8\% na densidade dos indivíduos acima de $5 \mathrm{~cm}$ \\ de diâmetro. O crescimento diamétrico da espécie em três anos de observação foi de $0,37 \mathrm{~cm}$. Embora baixo ele é crescente, \\ justificado pela abertura do dossel. A análise da distribuição diamétrica mostra que a abertura do dossel foi benéfica para o \\ incremento diamétrico da espécie, aumentando o número de indivíduos em cinco das oito classes de diâmetro analisadas, \\ mantendo-se ainda como uma distribuição decrescente.
}

PALAVRAS-CHAVE: Sterculia pruriens, crescimento diamétrico, monitoramento, exploração florestal

\section{Behavior of Sterculia pruriens (Aubl.) Schum. Sterculiaceae (axixá), in a managed tropical forest, Moju - PA}

\section{ABSTRACT}

This study aims to evaluate the diameter growth of the Sterculia pruriens (Aublet) Schumann - Sterculiaceae (axixá) population, after selective logging. The study was carried out in the Embrapa Amazônia Oriental Experimental Station in the Moju municipality, State of Pará - Brazil, situated between the coordinates $02^{\circ} 07^{\prime} 30^{\prime \prime}$ and 2० $12^{\prime} 06^{\prime \prime}$ of South Latitude and $48^{\circ}$ $46^{\prime} 57^{\prime \prime}$ and $48^{\circ} 48^{\prime} 30^{\prime \prime}$ of West Longitude. In each of nine selected gaps, $10 \mathrm{~m}$ x $50 \mathrm{~m}$ strips were installed, starting from the gap boarder into the forest interior, in North, South, East and West directions. Each strip was divided into 10m square plots, numbered from 1 to 5, constituting sampling level II. We analyzed the species diameter distribution with an amplitude of more or equal to $5 \mathrm{~cm} \mathrm{dbh}$, up to the maximum value of $66,8 \mathrm{~cm}$ which was that measured in the beginning of the monitoring. In the study area, we found high density of Sterculia pruriens individuals immediately and three years after the forest exploitation. In the end of the study, we observed a $9.8 \%$ increase in density for individuals above $5 \mathrm{~cm}$ dbh. The diameter growth for the species in three years of monitoring was $0.27 \mathrm{~cm}$. Although low, it is increasing, because of the canopy opening. The diameter distribution analysis shows that the opening was beneficial for the diameter increment of the species, increasing the number of individuals in five of the eight diameter classes analyzed; however, it remains a decreasing distribution.

KEYWORDS: Sterculia pruriens, diameter growth, monitoring, logging

1 Universidade Federal Rural da Amazônia -UFRA, E-mail: fernando.jardim@ufra.edu.br

${ }^{2}$ Universidade Federal Rural da Amazônia - UFRA, E-mail: soaresufra@hotmail.com 


\section{INTRODUÇÃO}

$\mathrm{O}$ manejo florestal destinado à produção madeireira em uma base sustentável ainda representa um grande desafio, dada à complexidade do ecossistema da floresta tropical. Para que as florestas naturais possam ser utilizadas em bases sustentáveis, com a adoção da técnica do manejo florestal preconizada pela legislação florestal brasileira, é fundamental que se quantifique a capacidade de reposição dos estoques extraídos das espécies com mercado atual e daquelas com mercado potencial.

Silva (2000) explica que a grande diversidade de espécies florestais, principalmente na floresta amazônica, aliada a grande pressáo exercida pela exploraçáo desordenada e predatória dessa floresta, torna imperativo que estudos sejam realizados, buscando melhor atender e elucidar dentro dos aspectos formadores da estrutura florestal, aqueles que possam vir a subsidiar e garantir um manejo adequado da mesma. Os sistemas silviculturais propostos para o manejo das florestas naturais só serấo eficientes na manutençẫo da produtividade da floresta, se a extração das espécies for feita de modo responsável e houver um compromisso efetivo com a sustentabilidade dos recursos.

Para indicar um tratamento silvicultural adequado é necessário que se conheça o comportamento das espécies em diferentes ambientes da floresta, de acordo com suas necessidades de radiação. $\mathrm{O}$ conhecimento da dinâmica de crescimento das espécies de interesse na floresta precede a adoção de técnicas adequadas de manejo florestal.

O manejo florestal é considerado a forma mais adequada de utilizaçáo dos recursos da floresta amazônica, tanto por estar voltado para a sua conservaçáo, buscando minimizar os distúrbios ambientais, como por atender às necessidades de desenvolvimento social e econômico (Costa, 2000). Várias experiências com manejo de florestas nativas no Brasil tem sido divulgadas, podendo-se citar os trabalhos realizados por Silva (1989); Souza (1989); Higuchi \& Vieira (1990); Vieira \& Higuchi(1990); Higuchi et al.(1991); Carvalho(1992); Veríssimo et al. (1992); Almeida \& Uhl (1993); Barreto et al. (1993); Lopes(1993); Souza \& Jardim(1993); Yared \& Souza(1993); Jardim(1995); Silva et al.(1995); Vieira(1995); Holmes et al.(2002); Serrão et al.(2003); Freitas(2004); Frances(2006); Sist \& Ferreira(2007) e Azevedo et al.(2008).

O entendimento dos padróes de estrutura ecológica, dos processos e dos fatores que governam a dinâmica de populaçóes é fundamental para a formulação de modelos de manejo florestal em longo prazo (Aragão \& Almeida, 1997) e ainda para a inserção de novas espécies para compor o conjunto das espécies de interesse comercial e econômico. Além disso, a distribuição do número de indivíduos por classe de diâmetro fornece valiosa informaçáo sobre a estrutura dimensional da floresta, sendo bastante importante para a silvicultura (Freitas, 2004), podendo ainda informar a tendência de desenvolvimento do povoamento, da espécie ou grupos de espécies (Lamprecht, 1990; Freitas, 2004).

Poucos estudos realizados na Amazônia abordaram o comportamento das espécies arbóreas e apresentaram estimativas de crescimento diamétrico dessas espécies em condiçôes de floresta natural (Jonkers, 1987; Silva, 1989; Jardim, 1990; Riera, 1990; Carvalho, 1992, Jardim et al. 1996a).

Este trabalho teve como objetivo avaliar o incremento diamétrico de árvores com DAP maior ou igual a $5 \mathrm{~cm}$, de Sterculia pruriens (axixá), uma espécie madeireira de grande importância no Estado do Pará, em diferentes condiçôes ambientais, em clareiras abertas pela exploraçáo florestal seletiva, gerando subsídios para a silvicultura e o manejo da espécie, além de identificar o grupo ecológico ao qual a espécie pertence.

\section{MATERIAIS E MÉTODOS}

O estudo foi realizado na Estação Experimental da Embrapa-Amazônia Oriental no município de Moju, Estado do Pará, entre as coordenadas geográficas $02^{\circ} 07^{\prime} 30^{\prime \prime}$

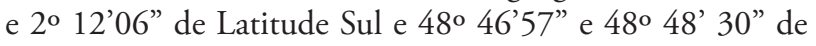
Longitude a Oeste do meridiano de Greenwich. Nessa área foi feita uma exploração florestal seletiva, realizada pela empresa Perachi Ltda., sob orientação da Embrapa Amazônia Oriental, em outubro de 1997, onde foram provocadas clareiras de vários tamanhos, das quais nove, com tamanho variando entre $231 \mathrm{~m}^{2}$ e $748 \mathrm{~m}^{2}$, foram selecionadas para monitoramento da vegetação com $\mathrm{DAP} \geq 5 \mathrm{~cm}$, da qual foi selecionado Sterculia pruriens para este estudo.

O clima da regiáo é do tipo Ami, segundo a classificação de Köppen. A temperatura média anual vai de $25^{\circ} \mathrm{C}$ a $27^{\circ} \mathrm{C}$. A precipitação pluviométrica anual varia de $2.000 \mathrm{~mm}$ a $3.000 \mathrm{~mm}$, com distribuição irregular, tendo pequeno período seco que vai de agosto a outubro. A insolaçáo mensal varia entre 148 , $0 \mathrm{~h}$ e 275 , $8 \mathrm{~h}$ e os valores mais elevados ocorrem no período de junho a dezembro, apresentando estreita relação com a precipitação (Nemer \& Jardim, 2004).

Em cada uma das nove clareiras selecionadas foram instaladas faixas de $10 \mathrm{~m} \times 50 \mathrm{~m}$, começando na bordadura da clareira para o interior da floresta, nas direçóes Norte, Sul, Este e Oeste. Cada faixa foi dividida em parcelas quadradas de $10 \mathrm{~m}$ de lado, que foram numeradas de 1 a 5 , da borda da clareira para o interior da floresta(Figura 1), para avaliar a influência das distâncias do centro das clareiras sobre o crescimento diamétrico das mesmas e onde foram medidas as árvores com DAP igual ou maior a $5 \mathrm{~cm}$. Após a instalação das parcelas, foi feita a identificação dos indivíduos de Sterculia pruriens. Em seguida foi feita a primeira medição da CAP (circunferência a 1,30 $\mathrm{m}$ do solo) com uma fita métrica. 


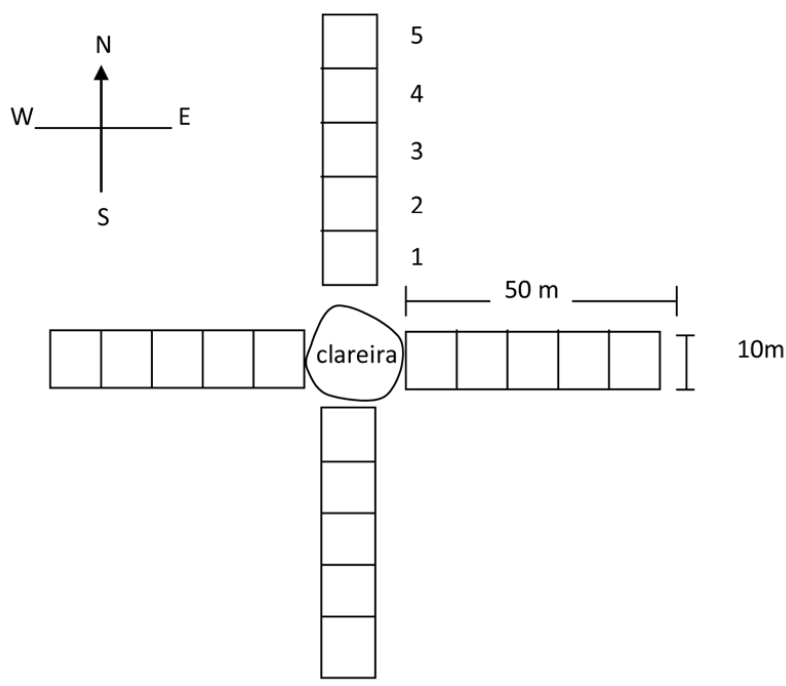

Figura 1- Desenho esquemático das parcelas amostrais para monitoramento dos indivíduos com DAP $\geq 5 \mathrm{~cm}$ em área de floresta densa de terra-firme, em Moju-PA.

A partir da medição de base, a cada três meses e ao final de cada estação do ano, foram feitas mediçóes, as quais começaram em junho de 1998 e terminaram em junho de 2001e pretendiam identificar possíveis estaçóes de crescimento das espécies madeireiras.

Foi analisada a distribuição diamétrica da espécie com amplitude de DAP $\geq 5 \mathrm{~cm}$ em intervalos de $5 \mathrm{~cm}$ de diâmetro, até o valor máximo de $66,8 \mathrm{~cm}$ encontrado no inicio do monitoramento, imediatamente após a exploração florestal, e no período de três anos de estudo após a exploraçáo. $\mathrm{O}$ crescimento foi analisado através do incremento corrente anual e trimestral em diâmetro, em um total de 32 árvores da espécie, distribuídas nas quatro direçôes em relação ao centro das clareiras.

Utilizou-se um delineamento inteiramente casualizado para testar a hipótese de que as direçôes influenciam na taxa de crescimento da espécie. Devido ao diferente número de árvores em cada direção, a comparaçáo dos resultados foi feita através do teste de Scheffé, a 95\% de probabilidade.

\section{RESULTADOS E DISCUSSÃO}

Na primeira medição do monitoramento, imediatamente após a exploração florestal seletiva, a espécie mostrou uma distribuição diamétrica contínua e decrescente até a classe diamétrica $9(\mathrm{CD} 9=45 \mathrm{~cm} \leq \mathrm{DAP}<50 \mathrm{~cm})$, do tipo "J" invertido. Aos trinta e seis meses de observação a espécie ainda mantém uma distribuição diamétrica decrescente, porém de forma descontínua, com o crescimento de indivíduos das classes de diâmetro 1, 2, 4, 6, 7 e 13 para as classes 2, 3, 5, 7, 8 e 14 respectivamente, e ausência de indivíduos em algumas classes (Figura 2). Uma distribuição de árvores desse tipo indica que a espécie é fortemente tolerante e pode regenerar sob o dossel, crescendo até a maturidade (Freitas, 2004) e formando uma associação estável (Jia-Rong \& Yang, 2007).

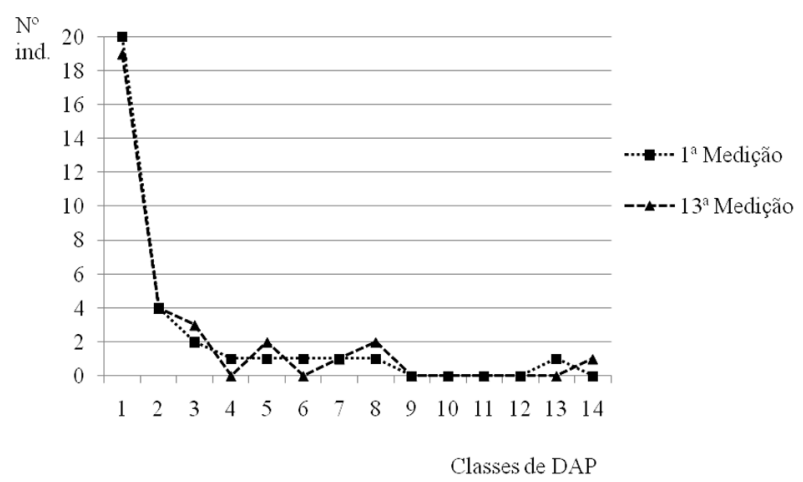

Figura 2 - Distribuição diamétrica de Sterculia pruriens (Aublet) Schumann (axixá) com DAP $\geq 5 \mathrm{~cm}$, na $1^{\mathrm{a}}$ e na $13^{\mathrm{a}}$ medição do monitoramento trimestral, em área de floresta explorada seletivamente no município de Moju-PA.

$\mathrm{Na}$ floresta natural, as espécies com distribuição na forma decrescente e contínua são aquelas chamadas tolerantes. As espécies com ausência de indivíduos nas classes de tamanho menores ou distribuição descontínua são chamadas secundárias e são fortemente heliófilas. As espécies cuja distribuiçáo diamétrica tem forma intermediária entre esses extremos são oportunistas, podendo ser de grandes ou pequenas clareiras, conforme demandem mais ou menos luz para se estabelecerem (Jardim et al., 1996b).

Maciel et al. (2000), estudando os parâmetros fitossociológicos de uma floresta tropical de terra firme na Floresta Nacional de Caxiuanã, também encontrou a distribuição diamétrica de algumas espécies seguindo a clássica distribuição "J" invertido. Esses resultados assemelham-se aos encontrados por Barros (1980); Carvalho (1992); Ribeiro et al. (1999) e Barros et al. (2000).

A distribuição diamétrica realizada após 36 meses de monitoramento mostra que a abertura do dossel foi benéfica para o incremento diamétrico da espécie, explicada pela mudança de classes de alguns indivíduos em cinco das quatorze classes de diâmetro. As espécies tolerantes, apesar de se desenvolverem em condiçôes de dossel fechado e sub-bosque, beneficiam-se de radiação solar proveniente de clareiras, assim como dos chamados "sunflecks", pequenas nesgas de luz direta que atravessam o dossel florestal e atingem o solo (FREEDEN e FIELD,1991).

O incremento diamétrico de S.pruriens foi maior na borda das clareiras do que nas demais posiçôes, ao longo de todo o período de estudo (Figura 3). Durante esses três anos, os indivíduos da espécie, nessa posição, tiveram crescimento 
diamétrico total de 1,7 cm. Embora possua características de espécie tolerante, S.pruriens mostrou um comportamento oscilante nas cinco diferentes posiçóes das parcelas, tendo na borda, próximo do centro da clareira, o melhor incremento em diâmetro (Figura 3).

O incremento diamétrico de $\boldsymbol{S}$.pruriens foi menor a $20 \mathrm{~m}$, onde se pode observar que nos três períodos avaliados houve um incremento total de $0,4 \mathrm{~cm}$. Por outro lado, foi verificado que o incremento diamétrico, em geral, náo diminuiu com o passar do tempo (Figura 3).

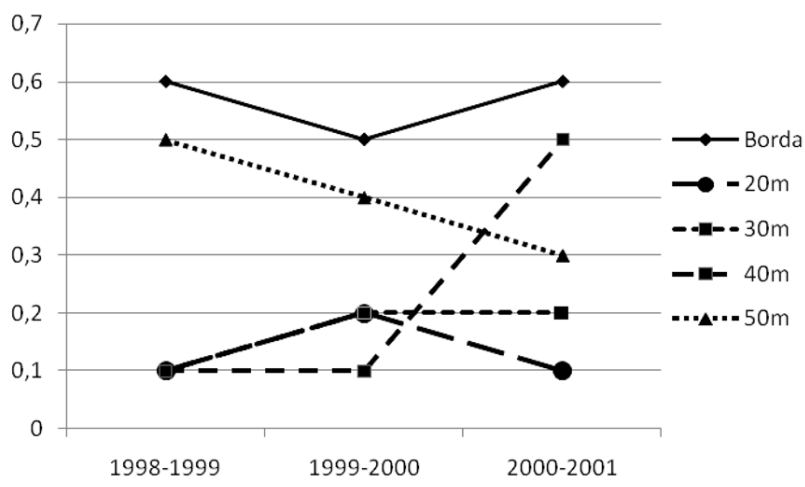

Figura 3 - Incremento corrente anual diamétrico (ICA)(cm), de Sterculia pruriens (Aublet) Schumann (axixá) em relação às distâncias do centro da clareira para dentro da floresta, em três anos de observação, em Moju-Pará.

Não houve indícios de influência das distâncias sobre o crescimento, uma vez que os indivíduos mais próximos do centro da clareira não foram os que mais cresceram, assim como aqueles mais distantes da clareira não foram os que apresentaram o menor incremento, como era de se esperar que acontecesse. A populaçáo de $S$. pruriens com DAP $\geq 5 \mathrm{~cm}$ mostrou variação em seu crescimento diamétrico nas cinco diferentes posiçôes das parcelas em torno das clareiras. Portanto, pode-se dizer que S.pruriens, apresenta características de espécie tolerante, mas beneficia-se com a formação de clareiras. Além disso, considerando a baixa mortalidade (Sena et al.,2000; Serrão,2001), é adaptável a condiçôes de luz menos propícias para seu desenvolvimento. No entanto, é preciso aprofundar os estudos sobre outros fatores ecofisiológicos.

De maneira geral, o incremento corrente anual de $S$. pruriens manteve-se relativamente constante ao longo do período de estudo, apesar de haver um ligeiro declínio no segundo ano de observaçáo, talvez devido ao adensamento da vegetação das clareiras, o que levou à competição por nutrientes, água e luz com outras espécies, nesse período (Figura 4).
ICA $(\mathrm{cm})$

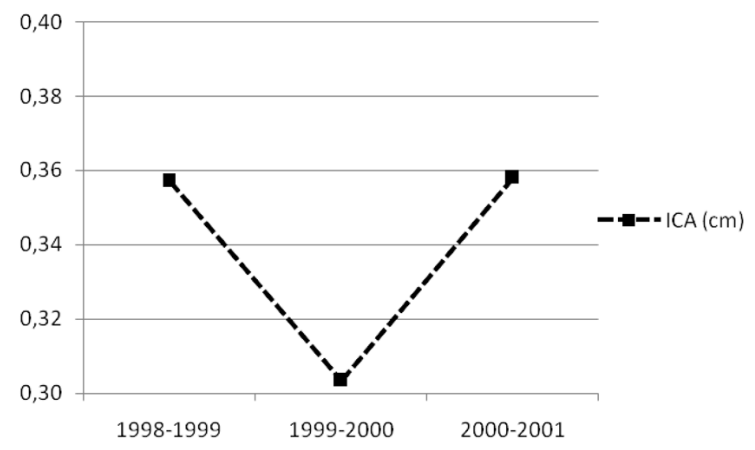

Figura 4 - Incremento Corrente Anual em diâmetro (cm) de Sterculia pruriens (Aublet) Schumann (axixá), durante três anos de observação, em Moju-Pará.

Não houve influências significativas, ao nível de 95\% de probabilidade, das diferentes direçóes no crescimento diamétrico de $\boldsymbol{S}$. pruriens(Tabela 1), em nenhum período do monitoramento. Todavia, o maior crescimento acumulado ocorreu na direção Sul das clareiras, com valor de $1,46 \mathrm{~cm}$ ao final do período de três anos de observaçóes, sendo que, no segundo ano (1999-2000), os indivíduos tiveram o menor incremento $(0,41 \mathrm{~cm})$. Na direçáo Norte, o crescimento acumulado em diâmetro dos indivíduos foi menor, com $1,05 \mathrm{~cm}$ durante o estudo (Figura 5). Nesse mesmo local, durante dois períodos de mediçôes (abril e outubro de 2000), a direção Sul foi a que mais recebeu radiação fotossinteticamente ativa, enquanto que a direção Norte foi a que recebeu menos (Malheiros, 2001).

Nas direções Este e Oeste o crescimento acumulado foi baixo comparado às direções Norte-Sul. A partir do segundo período ocorreu uma diferenciação, pois ambas sofrerem um declínio em seus incrementos e essa diminuição foi mais intensa na direçáo Este, agravando-se ainda mais no terceiro período, chegando a estagnar o incremento neste período. Já a direçáo Oeste retomou um incremento positivo no terceiro

ICA

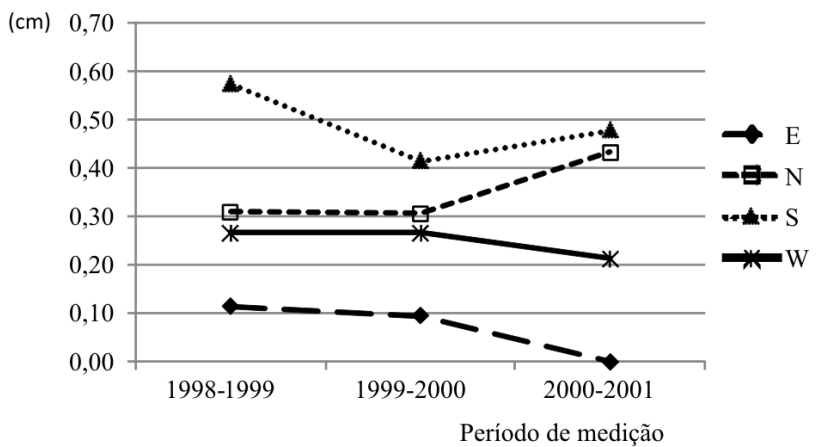

Figura 5 - Incremento corrente anual em diâmetro de Sterculia pruriens (Aublet) Schumann (axixá) nas direções Norte, Sul, Este e Oeste das clareiras, em três anos de observação, em Moju-Pará. 
Tabela 1 - Anova para Incremento Corrente Anual em diâmetro de Sterculia pruriens em função das direções N, S, E, e 0, em floresta tropical, no primeiro, no segundo e no terceiro períodos e para o Incremento Periódico três anos após a exploração, em Moju - PA

\begin{tabular}{|c|c|c|c|c|c|}
\hline Período & Fonte & $\mathrm{Gl}$ & $S Q$ & $M Q$ & F Calculado \\
\hline & Direções & 3 & 0,82 & 0,27 & $0,69 \mathrm{NS}$ \\
\hline \multirow[t]{3}{*}{ 1998-1999 } & Resíduo & 28 & 11,06 & 0,40 & \\
\hline & Total & 31 & 11,88 & & \\
\hline & Direções & 3 & 0,34 & 0,11 & $0,34 N S$ \\
\hline \multirow[t]{3}{*}{$1999-2000$} & Resíduo & 28 & 9,51 & 0,34 & \\
\hline & Total & 31 & 9,85 & & \\
\hline & Direções & 3 & 0,92 & 0,31 & $0,92 \mathrm{NS}$ \\
\hline \multirow[t]{3}{*}{$2000-2001$} & Resíduo & 28 & 9,41 & 0,34 & \\
\hline & Total & 31 & 10,33 & & \\
\hline & Direções & 3 & 5,49 & 1,83 & $0,65 \mathrm{NS}$ \\
\hline \multirow[t]{2}{*}{$1999-2001$} & Resíduo & 28 & 78,82 & 2,81 & \\
\hline & Total & 31 & 84,31 & & \\
\hline
\end{tabular}

$\mathrm{F}_{(0,05) 3,31}=2,92 ; \mathrm{F}_{(0,01) 3,31}=4,51$

período em relaçáo ao segundo período (Figura 5). Outros fatores como as diferentes fases de crescimento ou condiçóes de competição, no entanto, podem também ter influenciado esses resultados e devem ser investigados.

O crescimento diamétrico de Sterculia pruriens ao final de três anos de observação, representou um aumento de $1,1 \mathrm{~cm}$ no diâmetro, resultando uma média de $0,37 \mathrm{~cm}$ por ano (Figura 6), podendo ser comparado com Eschweilera odora $(0,33 \mathrm{~cm})$ (Nemer; Jardim, 2004) e Lecythis idatimon $(0,24 \mathrm{~cm})$ (Nascimento, 2003), na mesma área de estudo. Esses valores são considerados baixos quando comparados com outras espécies de rápido crescimento como Jacaranda copaia, que cresceu em diâmetro $0,79 \mathrm{~cm} / \mathrm{ano}$ em uma área explorada e $0,77 \mathrm{~cm} /$ ano em uma área não explorada, em 7 anos de estudo (Carvalho, 1992). Todavia, Alder et al. (2002) classifica como médios, valores de crescimento diamétrico entre $0,3 \mathrm{~cm}$ e $0,5 \mathrm{~cm}$. Serrão (2001) observou crescimento de $0,4 \mathrm{~cm}$ em diâmetro de base do caule e de $58,2 \mathrm{~cm}$ em altura para Jacaranda copaia, por ano, em dois anos de observação, nessa mesma área em Moju. Azevedo et al.(2008) encontraram Incremento Periódico Anual(IPA) em diâmetro variando de $0,38 \mathrm{~cm} \cdot$ ano $^{-1}$ para povoamento comercial e de $0,30 \mathrm{~cm} \cdot \mathrm{ano}^{-1}$ para povoamento total bruto.

Carvalho (1992) cita que as árvores de pequeno porte, que geralmente estão localizadas no sub-bosque, apresentam crescimento lento, mesmo quando o povoamento encontrase em fase de crescimento acelerado. Oliveira-Filho et al. (1994) observaram diferença no crescimento de acordo com o tamanho dos indivíduos, trabalhando em floresta secundária. Segundo esses autores, Vochysia maxima Ducke apresentou

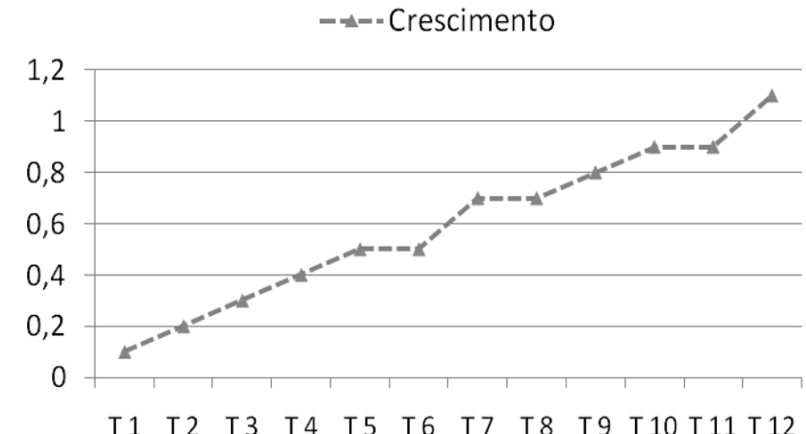

Figura 6 - Crescimento diamétrico acumulado de Sterculia pruriens (Aublet) Schumann - Sterculiaceae (axixá) durante três anos de observação, no Campo Experimental da Embrapa Amazônia Oriental no município de Moju-Pará.

crescimento de $1,46 \mathrm{~cm} /$ ano para árvores com DAP entre 5,0 e $9,9 \mathrm{~cm}$ e $2,11 \mathrm{~cm} /$ ano para árvores com diâmetros superiores a $60 \mathrm{~cm}$.

Sterculia pruriens não mostrou indícios de sazonalidade no crescimento diamétrico (Figura 7), embora mostre maior crescimento no período março/junho de 2000 e junho/ setembro de 2001. Todavia, Eschweilera odora na mesma área de estudo, mostrou uma sazonalidade no incremento com picos no período de setembro a dezembro (Nemer; Jardim, 2004). Esse autor cita ainda que no trimestre mais chuvoso (março/junho) a espécie apresenta um menor incremento, justificando de que os indivíduos no sub-bosque da floresta realizaram seu processo fotossintético com radiação menos intensa. 


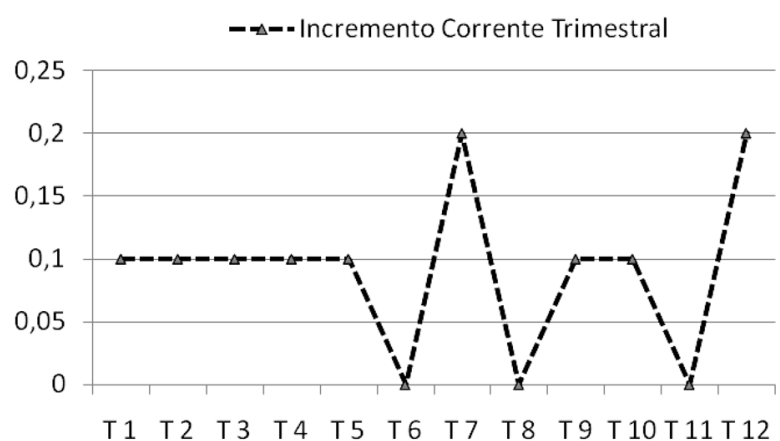

Figura 7 - Incremento corrente trimestral (cm) de Sterculia pruriens (Aublet) Schumann - Sterculiaceae (axixá) durante três anos de observação, no Campo Experimental da Embrapa Amazônia Oriental no município de Moju-Pará.

O beneficio da abertura do dossel gradativamente diminui à medida que o tempo passa. Fato semelhante foi observado em experimentos silviculturais no Suriname (Graaf et al., 1999). No Tapajós, a floresta desceu quase ao nível de uma floresta não explorada. Esse fato indica que o desbaste deveria ser prática silvicultural normal de manejo, visto que o aparecimento de clareiras aumenta consideravelmente a disponibilidade de luz. Todavia, manipular aberturas de dossel para obter florestas adequadas não é uma tarefa fácil em florestas tropicais devido à sua grande diversidade, onde uma mistura de espécies arbóreas de temperamentos diferentes co-ocorre e onde cada espécie responde diferentemente à abertura de dossel (Freitas, 2004) .

\section{CONCLUSÃO}

O incremento diamétrico dos indivíduos da população de Sterculia pruriens nos três anos avaliados em relação às distâncias do centro das clareiras para dentro da floresta e às direçóes Norte, Sul, Este e Oeste variou, mas não tendeu a diminuir.

Sterculia pruriens é uma espécie com estabilidade ecológica, mantendo-se com uma distribuição diamétrica decrescente após três anos da exploração florestal seletiva. Por essa razão, neste estudo, Sterculia. pruriens ficou classificada como uma espécie pertence ao grupo das tolerantes.

O crescimento diamétrico da espécie apresentou variação no período estudado, com média de $0,37 \mathrm{~cm}$ por ano, durante os três anos de observação. Esse resultado, aliado à forma da sua distribuição diamétrica contínua e decrescente, permitem projetar um ciclo de corte de 27 anos para o manejo da espécie em um sistema silvicultural policíclico.

\section{BIBLIOGRAFIA CITADA}

ALDER, D.; OAVIKA, F.; SANCHEZ, M.; SILVA, J.N.M.; VAN DER HOUT, P.; WRIGHT, H.L. 2002. A comparison of species rates from four moist tropical forest regions using increment-size ordination. The International Forestry Review, 4(3): 196-205.
ALMEIDA, T.; UHL, C. 1993. Developing a model for planning of the soil use in the Eastern Amazonia with a quantitative database: Paragominas case. IMAZON/EMBRAPA - CPATU. Belém, Pará. 30 p. (in Portuguese)

ARAGÃO, I. L. G.; ALMEIDA, S. S. 1997. Compared ecological structure of populations of acapu (Vouacapona americana Aubl. Caesalpiniaceae) in two terra firme forests of the Eastern Amazônia. In: LISBOA, P. L. B. (Eds). Caxiuanã. Museu Paraense Emílio Goeldi, Belém, Pará. p. 227-290.(in Portuguese)

AZEVEDO, C. P. de, SANQUETTA, C. R., SILVA,J. N. M., MACHADO, S. do A. 2008 Effect of logging and the silvicultural treatments on the ecological grouping of species. FLORESTA, 38(1): 53-69. (in Portuguese, with abstract in English)

BARRETO, P.G.B.; UHL, C.; YARED, J.A.G. 1993. The potential of wood sustainable production in Paragominas - Pará, in the Eastern Amazônia: ecological and economic accountig of. In: CONGRESSO FLORESTAL BRASILEIRO e CONGRESSO FLORESTAL PAN-AMERICANO, Curitiba, 1993. Anais. Curitiba, p. 387-392.(in Portuguese)

BARROS, P. L. C. de. 1980. Studies of the diameter distributions of the National Forest of the Tapajós - Pará. Dissertação de Mestrado, Universidade Federal do Paraná, Curitiba, Paraná. 123p. (in Portuguese)

BARROS, A.V. de; BARROS, P.L.C. de; SILVA, L.C.B. da 2000. Phytosociological analysis of a forest in Curuá-Una - Pará. Revista de Ciências Agrárias, 34: 9-36. (in Portuguese, with abstract in English)

CARVALHO, J.O.P. 1992. Structure and dynamics of a logged over Brazilian Amazonian rain forest. PhD Thesis, Oxford University. Oxford. 215p.

COSTA, D. H. M. 2000. Dynamics of the forest composition and growth of a terra firme forest area in the Flona Tapajos after wood harvest. Dissertação de Mestrado, Faculdade de Ciências Agrárias do Pará, Belém, Pará. 81p. (in Portuguese)

FRANCES, L. M. de B. 2006. Impact of the logging in the structure of a forest in the Paragominas region, Pará, in regard to two intensities of wood harvest. Dissertação de Mestrado, Universidade Federal Rural da Amazônia, Belém, Pará. 203p. (in Portuguese)

FREDEEN,A. L., FIELD,C. B. 1991. Leaf respiration in Piper species native to a Mexican rain forest. Physiologia Plantarum, n. 82 , p. 85-92.

FREITAS,J. V. de. 2004. Improving Tree Selection for Felling and Retention in Natural Forest in Amazonia Through Spatial Control and Targeted Seed Tree Retention: A Case Study of a Forest Management Project in Amazonas State, Brazil. PhD Thesis, University of Aberdeen, Aberdeen. 207p.

GRAAF, N.R. de; POELS, R.L.H.; VAN ROMPACY, R.S.A.R. 1999. Effect of silvicultural treatment on growth and mortality of rainforest in Suriname over long periods. Forest Ecology and Management, 124: 123-135.

HIGUCHI, N.; VIEIRA, G. 1990 Sustained management of terra firme tropical rain forest in the Manaus region - a research project of the INPA. In: CONGRESSO FLORESTAL BRASILEIRO, 
6, Campos do Jordão, 1990. Anais. Campos do Jordão, 1990. p. 34-37. (in Portuguese)

HIGUCHI, N.; VIEIRA, G.; MINETTE, L. J.; FREITAS, J. V.; JARDIM, F. C. S. 1991. Forest development: S.E.L. System (Selection of Listed Species) to manage the terra firme tropical rain forest of the Amazonia. In: Val, A.L., Figliuolo, R., Feldberg, E. (Eds.) Scientific bases for development and preservation strategies of the Amazonia: facts and perspectives. SCT/Instituto Nacional de Pesquisas da Amazônia. Manaus, Amazonas. p. 197-206 (in Portuguese)

HOLMES, T.P.; BLATE, G.M.; ZWEED, J.C.; PEREIRA JR.,R.; BARRETO, P.; BOLTZ, F.; BAUCH, R. 2002. Financial and ecological indicators of reduced impact logging performance in the eastern Amazon. Forest Ecology and Management, 163: 93-110.

JARDIM, F. C. da S. 1990. Mortality and growth in the equatorial terra firme forest. Boletim do Museu Paraense Emílio Goeldi, Série Botânica, 6(2): 227-234. (in Portuguese, with abstract in English)

JARDIM, F.C. da S.; SOUZA, A.L. de; SILVA, A.F. da; BARROS, N.F. de; SILVA, E.; MACHADO, C.C. 1996a. Dynamics of tree vegetation with $\mathrm{dbh}$ above $5 \mathrm{~cm}$ : comparison between functional and ecophysiological groups in the INPA's experimental station of tropical silviculture, Manaus-AM. Revista Árvore, 20(3): 267 278. (in Portuguese, with abstract in English)

JARDIM, F.C. da S.; SOUZA, A.L. de; BARROS, N.F. de; SILVA, E.; MACHADO, C.C.; SILVA, A.F. da. 1996b. Grouping of the tree species of an equatorial forest in the Manaus-AM region. Boletim da FCAP, 26: 7-29. (in Portuguese, with abstract in English)

JARDIM, F. C. da S. 1995. Behavior of natural regeneration of tree species in different intensities of thinning by girdling in the Manaus-AM region. Tese de Doutorado, Universidade Federal de Viçosa, Viçosa, Minas Gerais. 158p. (in Portuguese, with abstract in English)

JIA-RONG, G.; YANG, G. 2007. Effects of disturbance on regeneration of Abies fabri forests at the upper reaches of the Yangtze River. Forestry Studies in China, 9(1): 27-32.

JONKERS, W. B. J. 1987. Vegetation structure, logging damage and silviculture in a tropical rain forest in Suriname. Wageningen: Agricultural University, 172p. (Ecology and Management of Tropical Rain Forests in Suriname, 3).

LAMMPRECHT, H. 1990 Silviculture in the tropics: forest ecosystems and respective tree species, possibilities and sustained exploitation method. GTZ Eschborn, 1990. 343p. (in Portuguese)

LOPES, J. do C. A. 1993. Demography and temporal fluctuations of natural regeneration after logging: Flona do Tapajós-Pará. Dissertação de Mestrado, Universidade de São Paulo, Piracicaba, São Paulo. 133p. (in Portuguese)

MACIEL, M. de N.M.; QUEIROZ, W.T. de; OLIVEIRA, F. de. 2000. Phytosociological parameters of a terra firme tropical forest in the National Forest of Caxiuanã (PA). Revista de Ciências Agrárias, 34: 85- 106. (in Portuguese, with abstract in English)

MALHEIROS, M. A. B. 2001. Characterization of the flow of photosintetic active radiation, spectral irradiance and red/far red relation in gaps from the selective logging, in Moju-Pará, Brazil.
Dissertação de Mestrado, Faculdade de Ciências Agrárias do Pará. 93p. (in Portuguese, with abstract in English)

NASCIMENTO, Z. P. D. do. 2003. Population dynamics of Lecythis idatimon Aublet after selective logging in a terra firme tropical forest. Dissertação de Mestrado, Faculdade de Ciências Agrárias do Pará. 61p. (in Portuguese, with abstract in English).

NEMER, T. C., JARDIM, F.C. da S. 2004. An assesment of diameter growth rate in a population of Eschweilera odora (Poepp.) Miers with $\mathrm{dbh} \geq 5 \mathrm{~cm}$ in a logged tropical rainforest, Moju, state of Pará, Brazil. Revista de Ciências Agrárias, 41: 77-88. (in Portuguese, with abstract in English)

OLIVEIRA-FILHO, A.T.; SCOLFORO, J.R.S.; MELLO, J.M. 1994. Floristic composition and communitarian structure of a remainder of montana semideciduous forest in Lavras, MG. Revista Brasileira de Botânica, 17(2): 167-182. (in Portuguese, with abstract in English)

RIBEIRO, R.J.; HIGUCHI, N.; SANTOS, J dos; AZEVEDO, C.P. de. 1999. Plant structural analysis on Carajás and Marabá regions - PARA State, Brazil. Acta Amazônica, 29(2): 207-222. (in Portuguese, with abstract in English)

RIERA, B. 1990 The gaps: a model to the forest exploitation. In: ATELIERSURLAMENAGEMENTETLACONSERVATION DE L'ECOSYSTEME FORESTIER TROPICAL HUMIDE. Cayenne, 1990. Étude de cas, 33. (in French)

SENA, J. R. C. de et al. 2000. Natural regeneration dynamics of Sterculia pruriens (Aubl) Schum. Sterculiaceae (axixá), in gaps and their influenced areas in a selectively logged forest, Moju-Pará. In: SEMINÁRIO DE INICIAÇÃO CIENTÍFICA DA FCAP, SEMINÁRIO DE INICIAÇÃO CIENTÍFICA DA EMBRAPA AMAZÔNIA ORIENTAL 4. 2000, Belém. Resumos. FCAP. Unidade de Apoio à Pesquisa e a Pós-Graduação, Belém, Pará. p. 340-343. (in Portuguese).

SERRÃO, D.R. 2001. Growth and mortality of tree species, in gaps from the selective logging, in Moju-Pará, Brazil. Dissertação de Mestrado, Faculdade de Ciências Agrárias do Pará. 64p. (in Portuguese, with abstract in English).

SERRÃO, D.R. JARDIM, F.C. da S., NEMER, T.C. 2003. Survival of six forest species in a selectively logged area in Moju municipality, Pará. Cerne, 9(2): 153-163. (in Portuguese, with abstract in English).

SILVA, G. A. P. da. 2000. Evaluation of phytosociological behavior of the equatorial rain forest of the Experimental Station of Tropical Silviculture of the National Institute of Research of the AmazôniaInpa, Manaus-AM. Dissertação de Mestrado, Faculdade de Ciências Agrárias do Pará. 70p. (in Portuguese, with abstract in English).

SILVA, J.N.M. 1989. The behaviour of the tropical rain forest of the Brazilian Amazon after logging. PhD Thesis, University of Oxford, Oxford. 302 p.

SILVA, J.N.M; CARVALHO, J.O.P. de; LOPES, J. do C.A.; ALMEIDA, B.F. de; COSTA, D.H.M.; OLIVEIRA, L.C. de; VANCLAY, J.K. e SKOVSGAARD, J.P. 1995. Growth and yield of a tropical rain forest in the Brazilian Amazon 13 years after logging. Forest Ecology and Management, 71: 267-274. 
SIST, P.; FERREIRA,F.N. 2007. Sustainability of reduced-impact logging in the Eastern Amazon. Forest ecology and management, 243(2-3): 199-209.

SOUZA, A.L. de. 1989. Analysis multivaried for natural forests management: alternatives of wood sustained yield for saw. Tese de Doutorado, Universidade Federal do Paraná, Curitiba. 255 p. (in Portuguese).

SOUZA,A.L.de, JARDIM,F. C. S. 1993 Silvicultural systems applicable to the tropical florests. Viçosa, SIF. 125p. (Documento SIF, 008). (in Portuguese)

VERÍSSIMO, A.; BARRETO, P.; MATTOS, M.; TARIFA, R.; UHL, C. 1992. Logging impacts and prospects for sustainable forest management in an oriental Amazonian frontier: the case of Paragominas. Forest Ecology and Management, 55: 169-199.
VIEIRA, G. 1995 Dynamics of the remainning plant population in gaps after logging in the Amazon. Conference on Forestry and Forest Products Research. p. 54-67.

VIEIRA, G. \& HIGUCHI, N. 1990 Effect of the gap size on natural regeneration in mechanically logged forest in the Brazilian Amazonia. In: $6^{0}$ Congresso Florestal Brasileiro, Campos do Jordão, São Paulo, v. 3. P. 666-672. (in Portuguese).

YARED, J.A; SOUZA, A.L. 1993 Analysis of the environment impacts of the tropical forest management. Viçosa. UFV. 38 p. (Documento SIF, no 9). (in Portuguese).

Recebido em 17/09/2008

Aceito em 28/10/2009 